\title{
Primary Vaginal Invasive Squamous Cell Carcinoma: A Rare Case Report
}

Santosh $\mathrm{T}^{1^{*}}$, Pramita Sahu ${ }^{2}$, Manoj Kumar Patro ${ }^{2}$ and Bandana Mishra ${ }^{2}$

${ }^{1}$ Department of Pathology, Seth GSMC \& KEMH, Mumbai-12, India

${ }^{2}$ Department of Pathology, MKCG Medical College, Berhampur, Odisha, India

*Corresponding author: Santosh T, Fellow Cytopathology, Department of Pathology, Seth GSMC \& KEMH, Mumbai-12, India, Tel: 08895495670; E-mail: born_vss@yahoo.co.in

Received date: Feb 07, 2017; Accepted date: Apr 41, 2017; Published date: Apr 26, 2017

Copyright: ( 2016 Santosh T, et al. This is an open-access article distributed under the terms of the Creative Commons Attribution License, which permits unrestricted use, distribution, and reproduction in any medium, provided the original author and source are credited.

\begin{abstract}
Primary squamous cell carcinoma (SCC) of the vagina is a rare entity in gynecological oncology. The tumor should be considered as primary vaginal carcinoma when the cervix is not involved. We report a case of primary squamous cell carcinoma of the vagina in a 62-year-old lady by histopathology. Cervix, endometrium and ovaries were meticulously examined to rule out a primary in these organs. She was then treated by radiotherapy. The rarity of the tumor and extremely strict criteria for diagnosis of primary squamous cell carcinoma in vagina tempted us to report the case.
\end{abstract}

Keywords: Vaginal carcinoma; Primary squamous cell carcinoma of the vagina; VAIN

\section{Abbreviations}

DNA: Deoxyribonucleic acid; DES: Diethylstilbesterol; EBRT: External beam radiotherapy; FIGO: International Federation of Gynaecology and Obstetrics; HPV: Human papilloma virus; H\&E: Haematoxylin and eosin; OPD: Out-patient department; SCC: Squamous cell carcinoma; SIL: Squamous intraepithelial lesion; USG: Ultrasonography; VAIN: Vaginal intraepithelial neoplasia

\section{Introduction}

Primary vaginal SCC is extremely rare in occurrence $(\sim 0.6$ per 100,000 women per year). It constitutes approx. $2 \%$ of genital malignancies [1]. Primary SCC of vagina needs strict criteria for diagnosis because of its close proximity to cervix where SCC is very common. To be considered a primary tumor of the vagina the neoplasm must be located in the vagina, without clinical or histological evidence of the involvement of the cervix or vulva [2]. The exact etiology is unknown but there are some predisposing factors i.e., HPV infection, vaginal intraepithelial neoplasia, exposure to DES and radiation. Radiotherapy/surgery/combination of both is the most accepted treatment modalities that depends on the clinical stage, anatomical location and size of the lesion [3]. We report a case of primary vaginal SCC in a 62 year female for its rarity.

\section{Case Report}

A 62-year-old postmenopausal female presented to our OPD, MKCG Medical College, Berhampur, India with complaints of intermittent, painless vaginal bleeding associated with weight loss and weakness for last 2 months. She had been treated intermittently for vulvovaginitis for last 3-4 years, without any complete cure. Family/ past treatment history was not significant. General and systemic examination findings were normal except mild pallor. All routine investigations findings were normal except mild anemia. Per speculum examination revealed an ulcero-proliferative growth of $2 \times 2 \mathrm{~cm}$ in the upper third of vagina on its anterior and right lateral wall that bleeds on touch. Cervix was healthy and fornices were free (Figure 1a). USG revealed an atrophic uterus with normal endometrial thickness. VIA and colposcopy were normal. Cervical pap smear was reported as negative for intraepithelial lesion or malignancy. Following which incisional biopsy was performed.

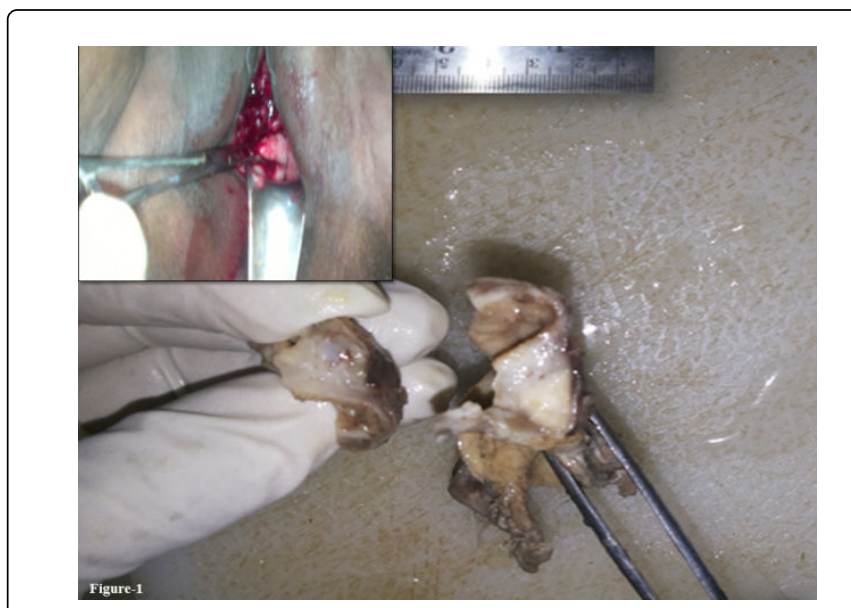

Figure1: Figure 1: Gross specimen showed grayish white illdefined tumor tissue, Per vaginum examination revealed an ulceroproliferative growth in the upper third of vagina on anterior and right lateral wall (Inset).

Grossly two soft tissue pieces of grayish white tissue of $1 \times 0.5 \times 0.5$ $\mathrm{cm}$ were received. Cut surface was solid, gray-white, friable with irregular margins and areas of hemorrhage (Figure 1). Microscopy showed stratified squamous epithelium with sub-epithelial tissue. Epithelium shows dysplastic changes, ulceration and loss of basement membrane with infiltration of pleomorphic tumor cells to the subepithelial tissue in sheets, nests and cords. Individual cell keratinization and abnormal mitotic figures were noted. Stroma was 
Page 2 of 3

desmoplastic with mononuclear inflammatory cell infiltration (Figure 2). A diagnosis of primary vaginal SCC-moderately differentiated grade was reported. The patient received external beam radiotherapy (EBRT) for 6 consecutive weeks. The lesion healed and on regular follow up, she was asymptomatic.

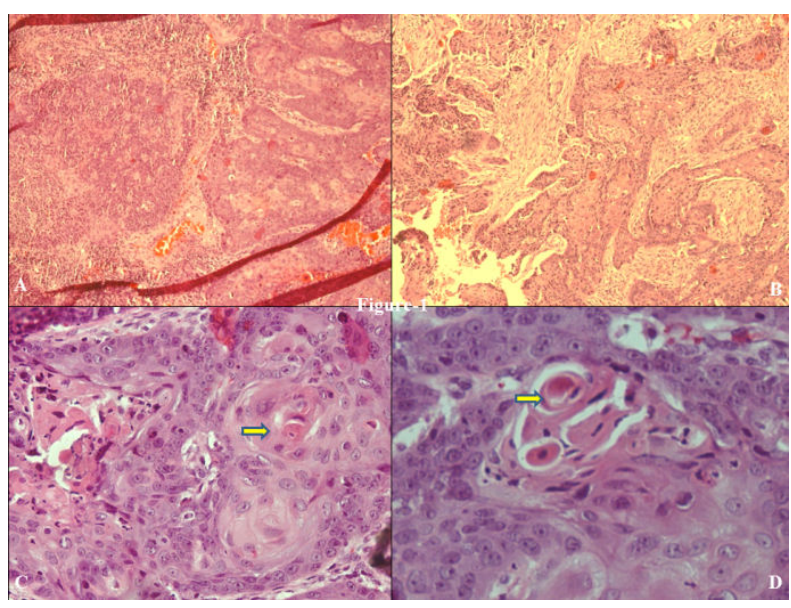

Figure 2: Vaginal SCC showing the presence of irregular nests of tumor cell with highly pleomorphic nuclei in sub epithelial stroma; Individual cell keratinization is also evident (arrow) (H\&E, $\times 10$ and $\times 40)$.

\section{Discussion}

Primary SCC of the vagina is a rare malignancy approximately accounting for $2 \%$ of all malignant tumors of the female genital tract [1]. Among vaginal malignancies, SCC is the commonest (95\%), others being adenocarcinoma, melanoma and sarcoma. Most vaginal SCCs are extensions of cervical or vulvar carcinomas. Exclusive vaginal tumors without clinical or histological evidence of the involvement of the cervix or vulva are classified as primary vaginal SCC. Bulky tumors located in the upper vagina with extension to the portio-vaginalis of the cervix and SCC vagina developing within 5 years of therapy for cervical carcinoma is not to be included considering them to be recurrent cervical carcinoma. Because of the strict criteria the incidence of primary vaginal squamous cell carcinoma restricts to only $10-20 \%$ of vaginal malignancies [2]. The risk factors for invasive squamous cell carcinoma of the vagina are the same as those for SIL (VAIN) those were prior vaginal discharge, condyloma acuminata or irritation, prior abnormal cervical/vaginal cytology, prior hysterectomy and rarely reported from surgically constructed neo-vaginas. Most of these patients are younger and developed carcinomas at least 8-25 years after the construction of a neo-vagina. Surprisingly early age at first intercourse, multiple sexual partners and a history of smoking is not associated with an elevated risk of the tumor. Almost all of the squamous cell carcinomas of the vagina are associated with high oncogenic risk HPV (i.e., 16, 18, 31, 33 and 35) genotypes detected by HPV-DNA sequencing [3-6]. The mean age for diagnosis of invasive vaginal SCC is 64 years [1]. Patients usually come with symptoms of painless vaginal bleeding or discharge, dysuria or frequency. Most primary vaginal carcinomas are grossly nodular, ulcerative, polypoid and fungating lesion. Size usually varies from clinically occult to $>10$ $\mathrm{cm}$. The upper $1 / 3^{\text {rd }}$ of the vagina with $57 \%$ involving the posterior wall and $27 \%$ the anterior/lateral wall is a common site [1]. Microscopically, SCCs of the vagina resemble those arising in cervix or vulva. Approximately $95 \%$ of vaginal SCC is of conventional type varying degrees of differentiation. The distinction of early invasive carcinoma from VAIN is based on a constellation of findings including the presence of angulated narrow cords of squamous cells at the stromal interface frequently with acquisition of more abundant eosinophilic cytoplasm and a desmoplastic or inflammatory host response [2].

The FIGO staging is one of the important prognostic indicators, others being tumor size and location (Table 1) [1].

\begin{tabular}{|l|l|l|}
\hline Stage & Clinical status & $\mathbf{5}$ \\
\hline I & The carvival & Year \\
\hline II & The carcinoma has involved the sub-vaginal tissue but has not extended to the pelvic wall & $53 \%$ \\
\hline III & The carcinoma has extended to the pelvic wall & $36 \%$ \\
\hline IV & $\begin{array}{l}\text { The carcinoma has extended beyond the true pelvis or has involved the mucosa of the bladder or rectum; bullous edema as such does } \\
\text { not permit a case to be allotted to Stage IV }\end{array}$ & \\
\hline IV a & Tumor invades bladder and/or rectal mucosa and/or direct extension beyond the true pelvis \\
\hline IV b & Spread to distant organs & \\
\hline
\end{tabular}

Table 1: FIGO staging of vaginal carcinoma (2012).

Prognosis is good for lesions in the upper $1 / 3^{\text {rd }}$ of the vagina Delayed initiation of therapy from the onset of symptoms may lead to early direct spread into the soft tissues of the pelvis, mucosa of the bladder or rectum as the wall of the vagina is thin and is separated from these organs by a thin connective tissue [2,5]. Lymphatic drainage of the vagina is complex with lesions in lower $2 / 3^{\text {rd }}$ usually metastasis to inguinal nodes whereas upper $1 / 3^{\text {rd }}$ lesions tend to spread to the iliac nodes. Metastases have also been documented in lungs and supraclavicular lymph nodes [1]. Most acceptable treatment modality for these cancers are a combination of EBRT and intra-cavitary radiation, local excision for small tumors and radical surgery for selected cases located in the upper third of vagina or posterior wall. Overall 5 and 10 year survival rates are $60 \%$ and $40 \%$ respectively in invasive SCCs. Recurrent disease bears an ominous prognosis with death within 1-2 years of diagnosis [6]. 
Citation: Santosh T, Sahu P, Manoj Kumar P, Bandana M (2017) Primary Vaginal Invasive Squamous Cell Carcinoma: A Rare Case Report. J

Page 3 of 3

\section{Conclusion}

Although primary vaginal SCCs is a rare malignant neoplasm of female genital tract, the incidence could not be ignored in a thickly populated country like India, since most of the victims are positive for high-risk HPV genotypes. Any fungating or ulcerative lesion in the vagina or decubitus ulcer not responding to treatment should be considered as malignant until proved otherwise.

\section{Ethics Approval}

All procedures performed in studies involving human participants were in accordance with the ethical standards of the institutional research committee.

\section{Consent for publication}

Written informed consent was obtained from the patient for publication of case report and accompanying images.

\section{Contributors}

TS carried out concepts and design, literature search, participated in the clinical study, data acquisition, data analysis and manuscript preparation will stand as guarantor also. MP carried out concepts and design, literature search. PS participated in clinical study and manuscript review. BM carried out literature search and data acquisition. All the authors have read and approved the final manuscript.

\section{References}

1. Begum N, Ara I, Islam F, Ganguly S, Afroj S (2012) Images in medical practices: primary vaginal carcinoma in prolapsed uterus. J Bangladesh Coll Phys Surg 30: 181-182.

2. Richard JZ, Marisa N, Kurman RJ (2011) Diseases of the Vagina. In: Kurman RJ, Ellenson LH, Ronnett BM, editors. Blaustein's Pathology of the Female Genital Tract, (6th edn). New York: Springer; pp: 135-137.

3. Shahana B, Rowshan A, Khadiza B (2012) Primary Vaginal Carcinoma, Tales of Tragedy: a Case report. Chattagram Maa-O-Shishu Hospital Medical College Journal 12: 65-67.

4. Andersen ES, Hanselaar AG, Paavonen J, Bergeron P (2003) Tumors of the vagina. In: Tavassoli FA, Devilee P, editors. World Health Organization Classification of Tumours. Pathology and Genetics of Tumours of the Breast and Female Genital Organs (4th edn). Lyon: IARC Press, pp: 292-95.

5. Mark HS, Mills SE (2010) The vulva and vagina. In: Mills SE, editor. Diagnostic Surgical Pathology( 6th edn). Philadelphia. Wolters Kulwer 21: $22-25$.

6. Juan Rosai (2011) Female reproductive system. In: Rosai J, editor. Rosai and Ackerman's Surgical Pathology (10th edn). China. Mosby Elsevier, pp: 1423-27. 